\title{
Constraining the range of Yukawa gravity interaction from S2 star orbits
}

\author{
D. Borka, ${ }^{a, 1}$ P. Jovanović, ${ }^{b}$ V. Borka Jovanović ${ }^{a}$ and A. F. \\ Zakharov ${ }^{c, d, e}$
}

${ }^{a}$ Atomic Physics Laboratory (040), Vinča Institute of Nuclear Sciences, University of Belgrade, P.O. Box 522, 11001 Belgrade, Serbia

${ }^{b}$ Astronomical Observatory, Volgina 7, 11060 Belgrade, Serbia

${ }^{c}$ Institute of Theoretical and Experimental Physics, B. Cheremushkinskaya 25, 117259 Moscow, Russia

${ }^{d}$ Bogoliubov Laboratory for Theoretical Physics, JINR, 141980 Dubna, Russia

${ }^{e}$ North Carolina Central University, 1801 Fayetteville Street, Durham, NC 27707, USA

E-mail: dusborka@vinca.rs, pjovanovic@aob.rs, vborka@vinca.rs, zakharov@itep.ru

\begin{abstract}
We consider possible signatures for Yukawa gravity within the Galactic Central Parsec, based on our analysis of the S2 star orbital precession around the massive compact dark object at the Galactic Centre, and on the comparisons between the simulated orbits in Yukawa gravity and two independent sets of observations. Our simulations resulted in strong constraints on the range of Yukawa interaction $\Lambda$ and showed that its most probable value in the case of S2 star is around 5000 - 7000 AU. At the same time, we were not able to obtain reliable constrains on the universal constant $\delta$ of Yukawa gravity, because the current observations of S2 star indicated that it may be highly correlated with parameter $\Lambda$ in the range $(0<\delta<1)$. For $\delta>2$ they are not correlated. However, the same universal constant which was successfully applied to clusters of galaxies and rotation curves of spiral galaxies $(\delta=1 / 3)$ also gives a satisfactory agreement with the observed orbital precession of the $\mathrm{S} 2$ star, and in that case the most probable value for the scale parameter is $\Lambda \approx 3000 \pm 1500 \mathrm{AU}$. Also, the Yukawa gravity potential induces precession of S2 star orbit in the same direction as General Relativity for $\delta>0$ and for $\delta<-1$, and in the opposite direction for $-1<\delta<0$. The future observations with advanced facilities, such as GRAVITY or/and European Extremely Large Telescope, are needed in order to verify these claims.
\end{abstract}

\footnotetext{
${ }^{1}$ Corresponding author.
} 


\section{Contents}

1 Introduction $\quad 1$

2 Simulated orbits of S2 star 3

$\begin{array}{lll}3 & \text { Fitting procedure } & 4\end{array}$

4 Results: simulations vs observations $\quad 6$

5 Conclusions 10

\section{Introduction}

The modified theories of gravity have been proposed like alternative approaches to Newtonian gravity on the ground of astrophysical and cosmological consequences of the observations of the Solar system, binary pulsars, spiral galaxies, clusters of galaxies and the large-scale structure of the Universe. The search for non-Newtonian gravity is part of the quest for non-Einsteinian physics which consists of searches for deviations from Special and General Relativity [1]. Different alternative gravity theories have been proposed (see e.g. [2-5] for reviews), such as: MOND [6-9], scalar-tensor [10], conformal [11, 12], Yukawa like corrected gravity theories [13-17] and extended theories of gravity $[4,5,18-22]$. One type of the extended theories of gravity is characterized by power-law Lagrangians [23, 24]. An extension of Post-Newtonian relativistic theory is presented by Kopeikin and Vlasov, who used a general class of the scalar-tensor (Brans-Dicke type) theories of gravitation [25]. Alternative approaches to Newtonian gravity in the framework of the weak field limit of fourth order gravity theory have been proposed and constraints on these theories have been discussed [2632]. Theories of "massive gravity" have also attracted some attention, and they could give an exponential decay to Newton's potential at the large distances (see e.g. [33] for a review). Babichev et al. introduced a new limit of that theory, in the weak-field approximation, which is able to capture both the Vainshtein recovery of general relativity and the large distance Yukawa decay [34]. For more details about massive gravity models see also the following papers [35-39].

So called Yukawa-like fifth force is a framework for deviations from the inverse-square law in which the gravitational potential deviates from the usual Newtonian form at large distances due to a Yukawa-like term in the gravitational potential [40-42]. Also it was proposed that the anomalous observations of the galactic rotation curves could be explained by addition of a Yukawa correction term to the Newtonian gravity potential [43]. In order to obtain the constraints on Yukawa gravity, studies of the planetary and stellar orbits at the larger scales, as well as laboratory searches at the smaller scales have to be performed. Adelberger et al. [44] reviewed experiments with very high sensitivity, placing constraints about new Yukawa forces from the exchange of very light scalar, pseudoscalar or vector particles. Weaker constraints at still smaller scales are available using the Casimir effect [see e.g. 14]. A compilation of experimental, geophysical and astronomical constraints on Yukawa violations of the gravitational inverse square law are given in Figs. 9 and 10 from [44] for different ranges. These results show that the Yukawa term is relatively well constrained for the short 
ranges (especially at sub-mm scale), but for long ranges further tests are needed, and it would be very important to evaluate parameters of the Yukawa law for whole range of $\Lambda$. A range around a few thousand $\mathrm{AU}$ has not been investigated yet. However, for longer distances Yukawa corrections have been successfully applied to clusters of galaxies setting $\delta=+1 / 3$ $[45,46]$. The same value of this parameter also gives a very good agreement between the theoretical and observational rotation curves of spiral galaxies [40].

For now it seems that the only major problem with Yukawa gravity is in the case of elliptical galaxies where the observations give a value of $\delta \approx-0.8$ [47], which is inconsistent with the one previously found for spiral galaxies. Napolitano et al. [47] found a possible explanation for this inconsistency, according to which $\delta$ might be correlated with the galaxy anisotropy and the scale parameter, where both elliptical and spiral galaxies follow the same pattern. In that case, $\delta$ could be interpreted in terms of physics of the gravitating systems after their spherical collapse [47].

On the other hand, S-stars are the bright stars which move around the centre of our Galaxy [48-53] where the compact radio source Sgr A* is located. These stars, together with recently discovered dense gas cloud falling towards the Galactic Centre [54], provide the most convincing evidence that Sgr A* represents a massive compact object around which S-stars are orbiting [53]. For one of them, called S2, there are some observational indications that its orbit could deviate from the Keplerian case due to relativistic precession [50, 55], but the current astrometric limit is not sufficient to unambiguously confirm such a claim.

The orbital precession can occur due to relativistic effects, resulting in a prograde pericentre shift or due to a possible extended mass distribution, producing a retrograde shift [56]. Both prograde relativistic and retrograde Newtonian pericentre shifts will result in rosette shaped orbits. Adkins and McDonnell [57] calculated the precession of Keplerian orbits under the influence of arbitrary central force perturbations. For some examples, including the Yukawa potential, they presented the results using hypergeometric functions. Weinberg et al. [58] discussed physical experiments achievable via the monitoring of stellar dynamics near the massive black hole at the Galactic Centre with a diffraction-limited, next-generation, extremely large telescope (ELT). They demonstrated that the lowest order relativistic effects, such as the prograde precession, could be detectable if the astrometric precision would reach a few tenths of mas. The astrometric limit for S2 star orbit today reaches 0.3 mas [59], and some very recent studies provide more and more evidence that orbit of S2 star is not closing (see e.g. Fig. 2 in [55]).

Here we study a possible application of Yukawa gravity within Galactic Central Parsec, for explaining the observed precession of orbits of S-stars. We assumed that the motion of these stars could be described by the gravitational potential around a massive compact central object (without speculating about its nature, i.e. whether it is a black hole or not), in any gravity theory which predicts a Yukawa correction term. For reviews of various theoretical frameworks yielding a Yukawa-like fifth force, such as braneworld models, scalar-tensor and scalar-tensor-vector theories of gravity, or studies of topological defects, see e.g. [60-64] and references therein. Other studies of long-range Yukawa-like modifications of gravity conducted with different astronomical techniques can be found in [41, 65-69]. Such a phenomenological approach is in some sense more general than studying the stellar orbits in a metric of a massive central black hole, since some classes of modified gravity theories (such as e.g. $f(R)$ theory) predict the black hole metrics which are equivalent to those obtained in General Relativity.

The present paper is organized as follows: in section 2 we describe our simulations of stellar orbits in Yukawa gravity potential; the procedure for fitting the simulated orbits to 
two independent sets of astrometric observations of $\mathrm{S} 2$ star is described in section 3; our main results are presented in section 4, and finally, we point out the most important conclusions of our studies in section 5 .

\section{Simulated orbits of S2 star}

As it was already mentioned, Yukawa gravity potential represents a widely used phenomenological approach to account for possible deviations from the Newtonian inverse-square law, by introducing the following exponential (or so called Yukawa-like) modification to the Newtonian gravitational potential [5, 43, 47]:

$$
\Phi(r)=-\frac{G M}{(1+\delta) r}\left[1+\delta e^{-\left(\frac{r}{\Lambda}\right)}\right],
$$

where $\Lambda$ is an arbitrary parameter (usually referred to as range of interaction), depending on the typical scale of the system under consideration and $\delta$ is a universal constant. For $\delta=0$ the Yukawa potential reduces to the Newtonian one, as expected.

We simulated orbits of S2 star in the Yukawa gravity potential (2.1) and compared the obtained results with two independent sets of observations of S2 star, obtained by New Technology Telescope/Very Large Telescope (NTT/VLT), as well as by Keck telescope (see Fig. 1 in [50]), which are publicly available as the supplementary on-line data to the electronic version of the paper [50]. The simulated orbits of S2 star were obtained in the standard way by numerical integration of differential equations of motion in Yukawa gravitational potential:

$$
\dot{\mathbf{r}}=\mathbf{v}, \quad \mu \ddot{\mathbf{r}}=-\nabla \Phi(\mathbf{r}),
$$

where $\mu$ is so called reduced mass in the two-body problem. In our calculations we assumed that the mass of central object is $M=4.3 \times 10^{6} M_{\odot}$ and that the distance to the S2 star is $d_{\star}=8.3 \mathrm{kpc}[50]$. Perturbations from other members of the stellar cluster, as well as from some possibly existing extended structures composed from visible or dark matter [29], were neglected due to simplicity reasons. The obtained simulated orbits in the Yukawa potential were compared with the two sets of observations of the S2 star. Since the integration of the equations (2.2) results with coordinates and velocity components in orbital plane (so called true orbits), the first step is to project them onto the observer's sky plane (i.e. to calculate the corresponding apparent orbits), in order to compare them with observed positions. From the theory of binary stars it is well known that any point $(x, y)$ on the true orbit could be projected into the point $\left(x^{c}, y^{c}\right)$ on the apparent orbit according to (see e.g.[70, 71]):

$$
x^{c}=l_{1} x+l_{2} y, \quad y^{c}=m_{1} x+m_{2} y,
$$

where the expressions for $l_{1}, l_{2}, m_{1}$ and $m_{2}$ depend on three orbital elements ( $\Omega$ - longitude of the ascending node, $\omega$ - longitude of pericenter and $i$ - inclination):

$$
\begin{aligned}
& l_{1}=\cos \Omega \cos \omega-\sin \Omega \sin \omega \cos i, \\
& l_{2}=-\cos \Omega \sin \omega-\sin \Omega \cos \omega \cos i, \\
& m_{1}=\sin \Omega \cos \omega+\cos \Omega \sin \omega \cos i, \\
& m_{2}=-\sin \Omega \sin \omega+\cos \Omega \cos \omega \cos i .
\end{aligned}
$$


Radial velocity can be calculated from the corresponding true position $(r, \theta)$ and orbital velocity $(\dot{r}, \dot{\theta})$ using the well known expression in polar coordinates [70]:

$$
v_{r a d}=\sin i[\sin (\theta+\omega) \cdot \dot{r}+r \cos (\theta+\omega) \cdot \dot{\theta}] .
$$

However, in our case it was more convenient to use the rectangular coordinates $x=r \cos \theta$ and $y=r \sin \theta$ to calculate the fitted radial velocities:

$$
\begin{aligned}
v_{\text {rad }}= & \frac{\sin i}{\sqrt{x^{2}+y^{2}}}[\sin (\theta+\omega) \cdot(x \dot{x}+y \dot{y})+ \\
& +\cos (\theta+\omega) \cdot(x \dot{y}-y \dot{x})],
\end{aligned}
$$

where $\theta=\arctan \frac{y}{x}$.

Since the present paper does not aim to study the Keplerian orbit of S2 star and since $i, \Omega$ and $\omega$ are needed here only for transforming from true to apparent coordinates, we used the following values obtained from the same observations [50]: $i=134^{\circ} .87, \Omega=226^{\circ} .53$ and $\omega=64^{\circ} .98$. One should also take into account that in the case of orbital precession $\omega$ is in general a function of time, and therefore it should be treated accordingly during the fitting procedure. However, based on some theoretical studies of precession in Yukawa gravity [7274] one can expect very slow change of $\omega$ during the observational interval of S2 star, and since in equations (2.4) $\omega$ is used only as an argument of sin and cos, fixing it to a constant value could introduce only negligible errors. Therefore, we assumed it together with $l_{1}, l_{2}, m_{1}$ and $m_{2}$ as constants when projecting true positions to their corresponding apparent values.

\section{$3 \quad$ Fitting procedure}

In that way, for each pair of a priori given values of $\delta$ and $\Lambda$, there are only four unknown parameters which are to be obtained by fitting: two components of initial position and two components of initial velocity in orbital plane, corresponding to the time of the first observation. We varied both $\delta$ and $\Lambda$, and for each pair of them found the best fit values of S2 star initial conditions and the corresponding value of $\chi^{2}$. The fitting itself was performed using LMDIF1 routine from MINPACK-1 Fortran 77 library which solves the nonlinear least squares problems by a modification of Marquardt-Levenberg algorithm [75]. Unfortunately, $\delta$ and $\Lambda$ could not be fitted simultaneously with initial position and velocity, most likely due to inability of the fitting routine to correctly estimate the corresponding components of Jacobian by a forward-difference approximation. Therefore, these two parameters were varied in certain domains, and their best fit estimates were found at the end of the fitting procedure as those for which the minimum $\chi^{2}$ was obtained.

We first adopted value of the universal constant $\delta=1 / 3$ and varied length scale $\Lambda$ in interval from 10 to $10000 \mathrm{AU}$ with increment of $10 \mathrm{AU}$, in order to see whether this value of $\delta$ could provide the satisfactory fit, as well as to estimate the most probable value of $\Lambda$ in this case. For each $\Lambda$ we obtained the best fit orbit using the following fitting procedure:

1. initial values for true position $\left(x_{0}, y_{0}\right)$ and orbital velocity $\left(\dot{x}_{0}, \dot{y}_{0}\right)$ of S2 star at the epoch of the first observation are specified; 

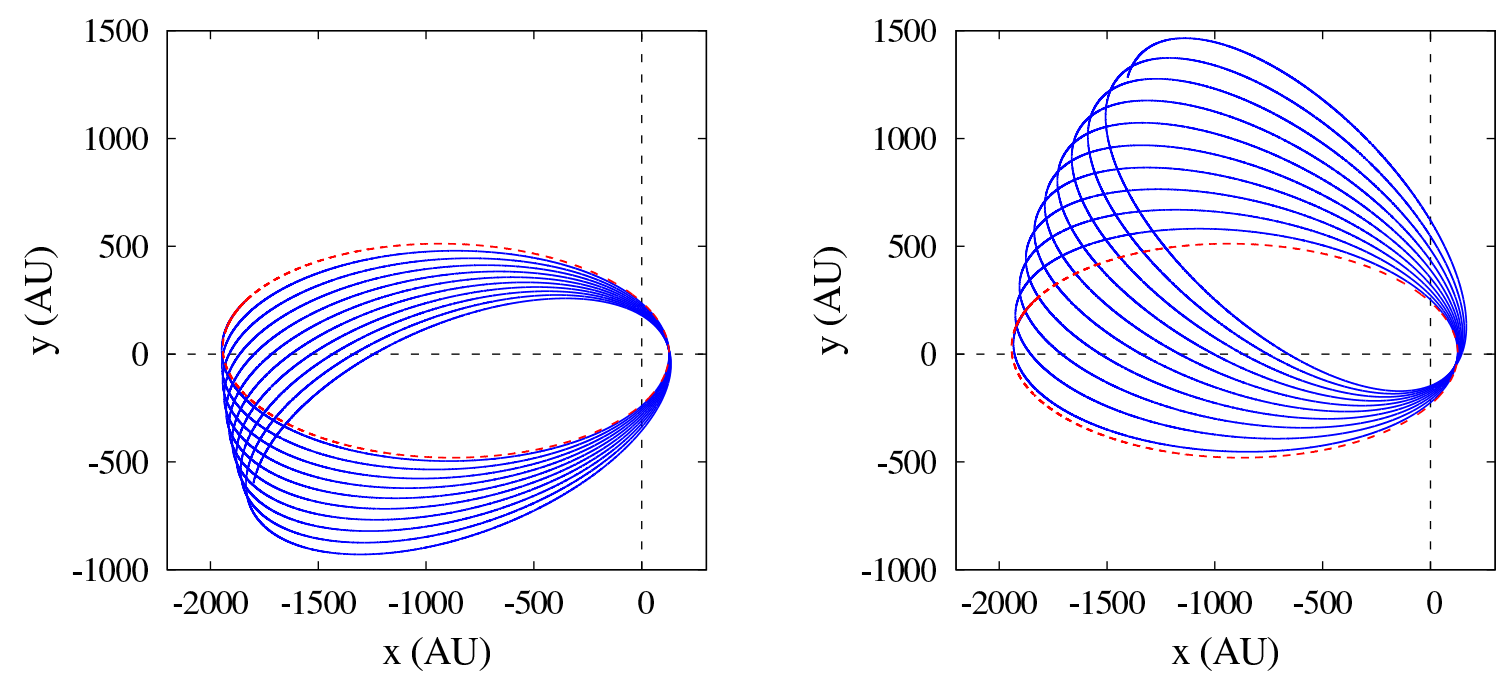

Figure 1. Comparisons between the orbit of S2 star in Newtonian gravity (red dashed line) and Yukawa gravity during 10 orbital periods (blue solid line) for $\Lambda=2.59 \times 10^{3} \mathrm{AU}$. In the left panel $\delta=+1 / 3$, and in the right $\delta=-1 / 3$.

2. the positions $\left(x_{i}, y_{i}\right)$ and velocities $\left(\dot{x}_{i}, \dot{y}_{i}\right)$ of S2 star along its true orbit are calculated for all observed epochs by numerical integration of equations of motion (2.2) in the Yukawa gravity potential;

3. the corresponding positions $\left(x_{i}^{c}, y_{i}^{c}\right)$ along the apparent orbit are calculated using the expressions (2.3) and (2.4), as well as the corresponding radial velocities $\left(v_{\text {rad }}^{i}\right)$ using $(2.6)$

4. the reduced $\chi^{2}$ of fit is estimated according the following expression:

$$
\chi^{2}=\frac{1}{2 N-\nu} \sum_{i=1}^{N}\left[\left(\frac{x_{i}^{o}-x_{i}^{c}}{\sigma_{x i}}\right)^{2}+\left(\frac{y_{i}^{o}-y_{i}^{c}}{\sigma_{y i}}\right)^{2}\right],
$$

where $\left(x_{i}^{o}, y_{i}^{o}\right)$ is the $i$-th observed position, $\left(x_{i}^{c}, y_{i}^{c}\right)$ is the corresponding calculated position, $N$ is the number of observations, $\nu$ is number of unknown parameters (in our case $\nu=4), \sigma_{x i}$ and $\sigma_{y i}$ are uncertainties of observed positions;

5. the reduced $\chi^{2}$ is minimized and the final values of initial positions and velocities are obtained.

Finally, we kept the value of $\Lambda$ which resulted with the smallest value of minimized reduced $\chi^{2}$.

In order to obtain some more general constraints on the parameters of Yukawa gravity, we also varied both $\delta$ and $\Lambda$ and studied the simulated orbits of S2 star which give at least the same or better fits than the Keplerian orbit. For each pair of these parameters the reduced $\chi^{2}$ of the best fit is obtained and used for generating the $\chi^{2}$ maps over the $\Lambda-\delta$ parameter space. These maps are then used to study the confidence regions in $\Lambda-\delta$ parameter space. 

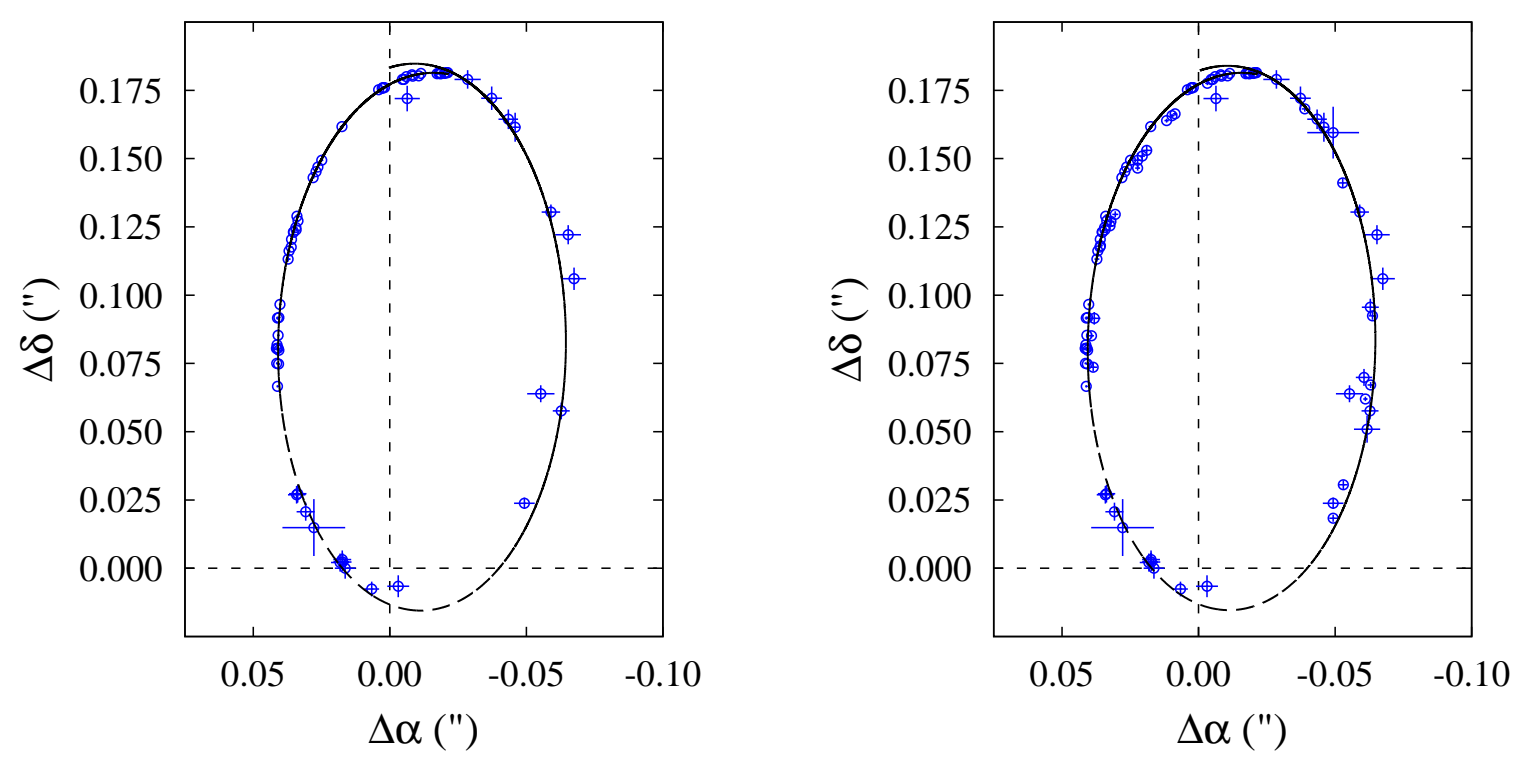

Figure 2. The fitted orbits in Yukawa gravity for $\delta=+1 / 3$ through the astrometric observations of S2 star (denoted by circles), obtained by NTT/VLT alone (left panel) and NTT/VLT + Keck (right panel). The best fits are obtained for $\Lambda=2.59 \times 10^{3} \mathrm{AU}$ and $\Lambda=3.03 \times 10^{3} \mathrm{AU}$, respectively.
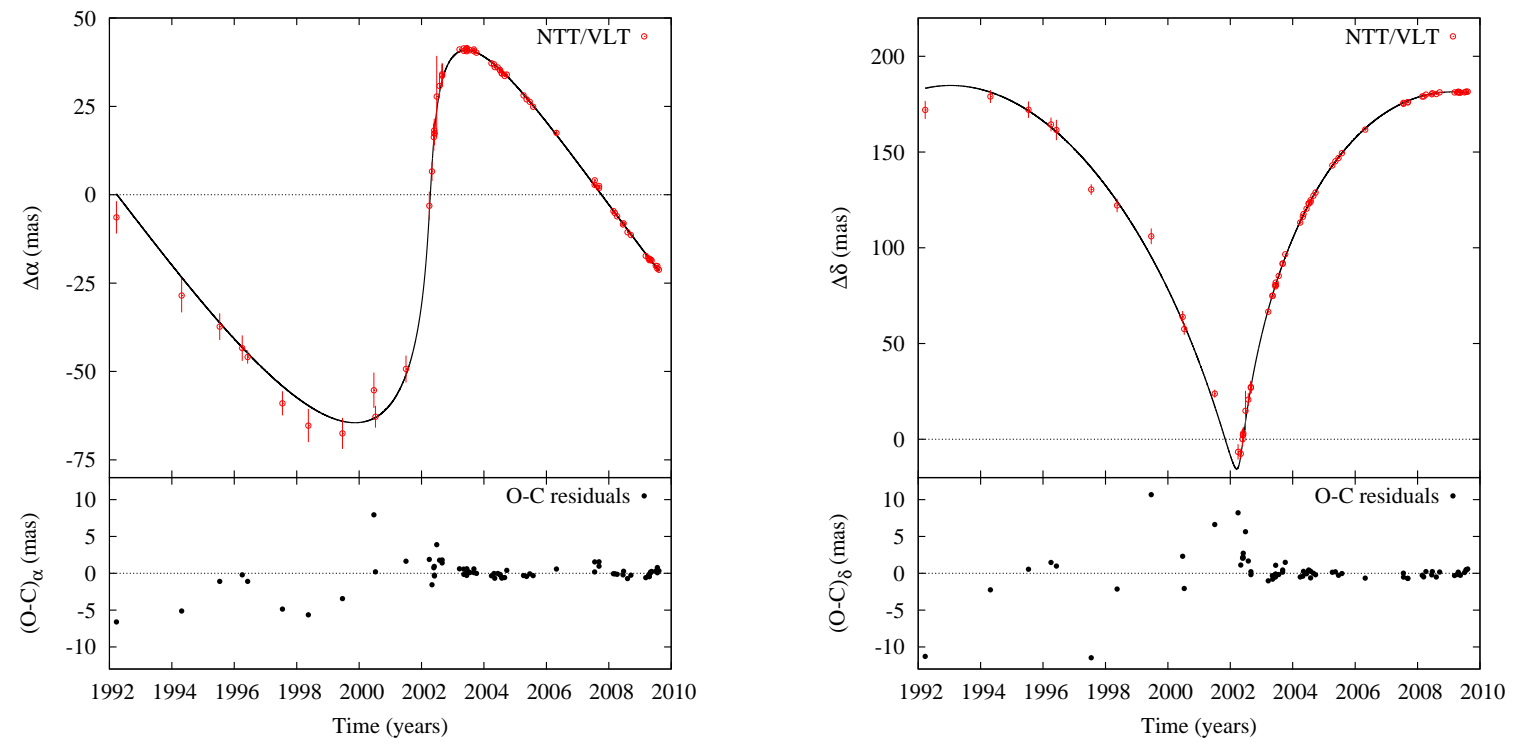

Figure 3. The comparisons between the observed (open circles with error bars) and fitted (solid lines) coordinates of S2 star (top), as well as the corresponding O-C residuals (bottom). The left panel shows the results for $\Delta \alpha$ and right panel for $\Delta \delta$ in the case of NTT/VLT observations and Yukawa gravity potential with $\delta=+1 / 3$ and $\Lambda=2.59 \times 10^{3}$ AU.

\section{Results: simulations vs observations}

The simulated orbits of S2 star around the central object in Yukawa gravity (blue solid line) and in Newtonian gravity (red dashed line) for $\Lambda=2.59 \times 10^{3} \mathrm{AU}$ and $\delta=+1 / 3$ (left panel) and $\delta=-1 / 3$ (right panel) during 10 periods, are presented in Fig. 1. We can notice that for $\delta=-1 / 3$ the precession has the negative direction and when $\delta=+1 / 3$ the precession has the 

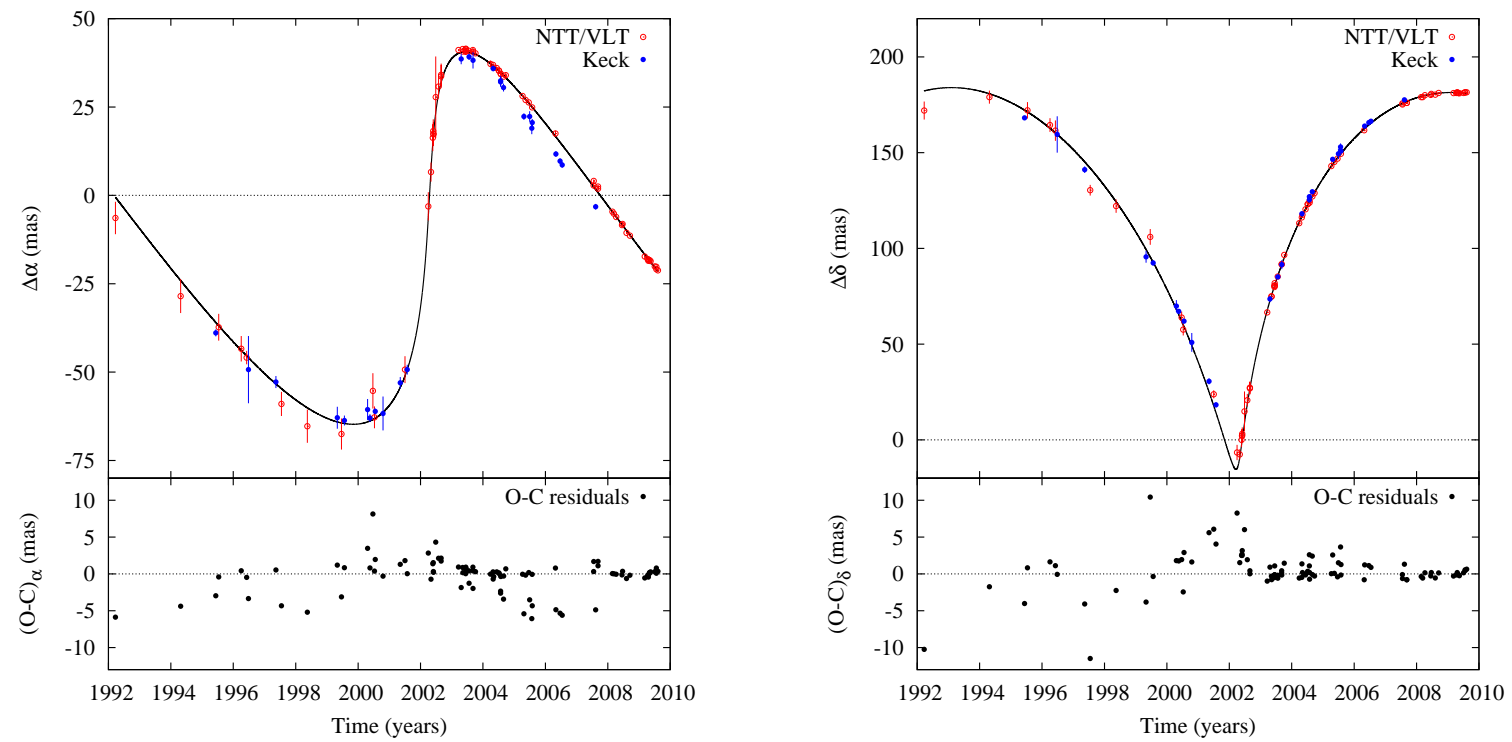

Figure 4. The same as in Fig. 3, but for NTT/VLT + Keck combined observations and for Yukawa gravity potential with $\Lambda=3.03 \times 10^{3} \mathrm{AU}$.
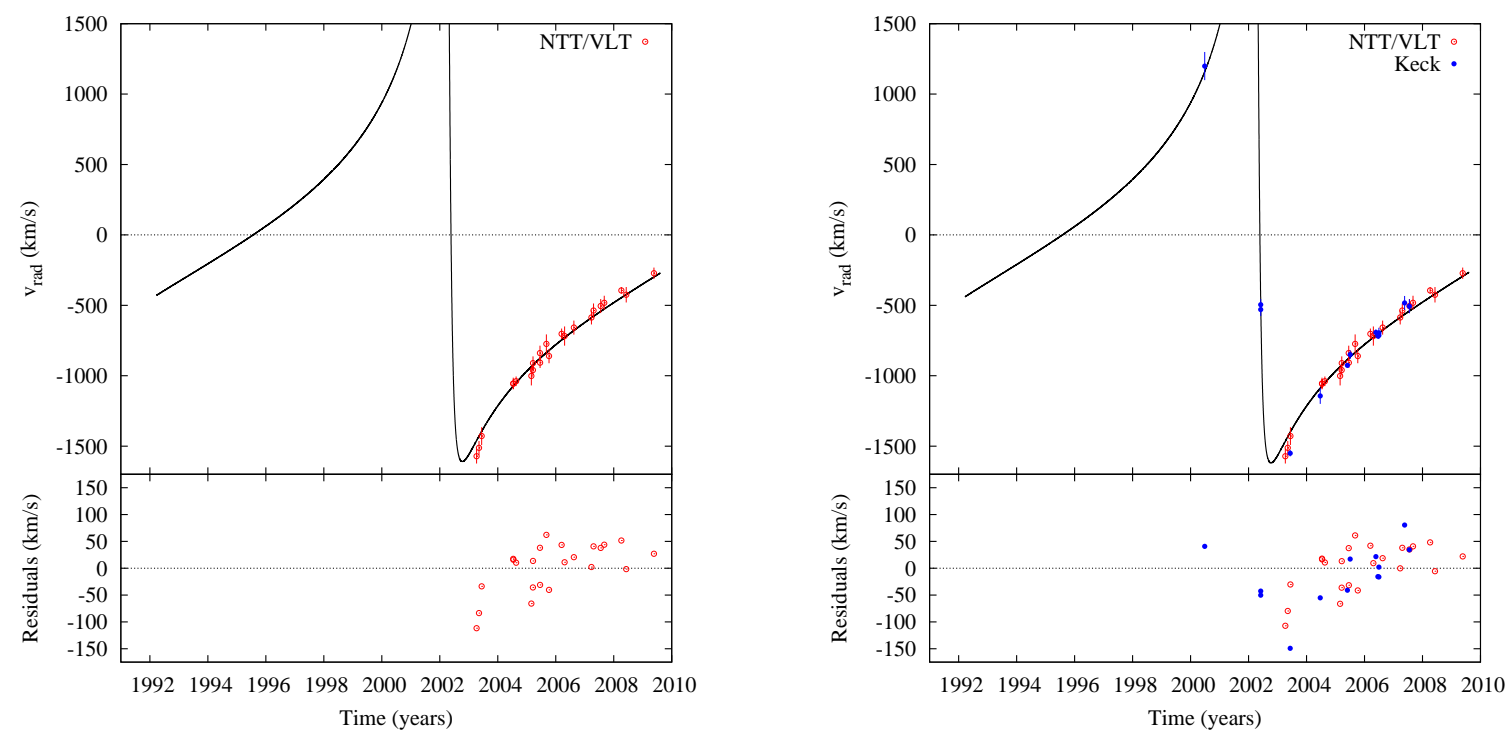

Figure 5. The comparisons between the observed (circles with error bars) and fitted (solid lines) radial velocities of S2 star (top), as well as the corresponding O-C residuals (bottom). The left panel shows the results in the case of NTT/VLT observations and Yukawa gravity potential with $\Lambda=2.59 \times 10^{3}$ $\mathrm{AU}$, while the right panel shows the results for $\mathrm{NTT} / \mathrm{VLT}+$ Keck combined observations and for Yukawa gravity potential with $\Lambda=3.03 \times 10^{3} \mathrm{AU}$. In both cases $\delta=+1 / 3$.

positive direction. Our analysis shows that the Yukawa gravity potential induces precession of S2 star orbit in the same direction as General Relativity for $\delta>0$ and for $\delta<-1$, and in the opposite direction for $-1<\delta<0$ as in the case of extended mass distribution or in $R^{n}$ gravity [26].

We used these simulated orbits to fit the observed orbits of S2 star. The best fit (according to NTT/VLT data) is obtained for the scale parameter: $\Lambda=2.59 \times 10^{3} \mathrm{AU}$, for 

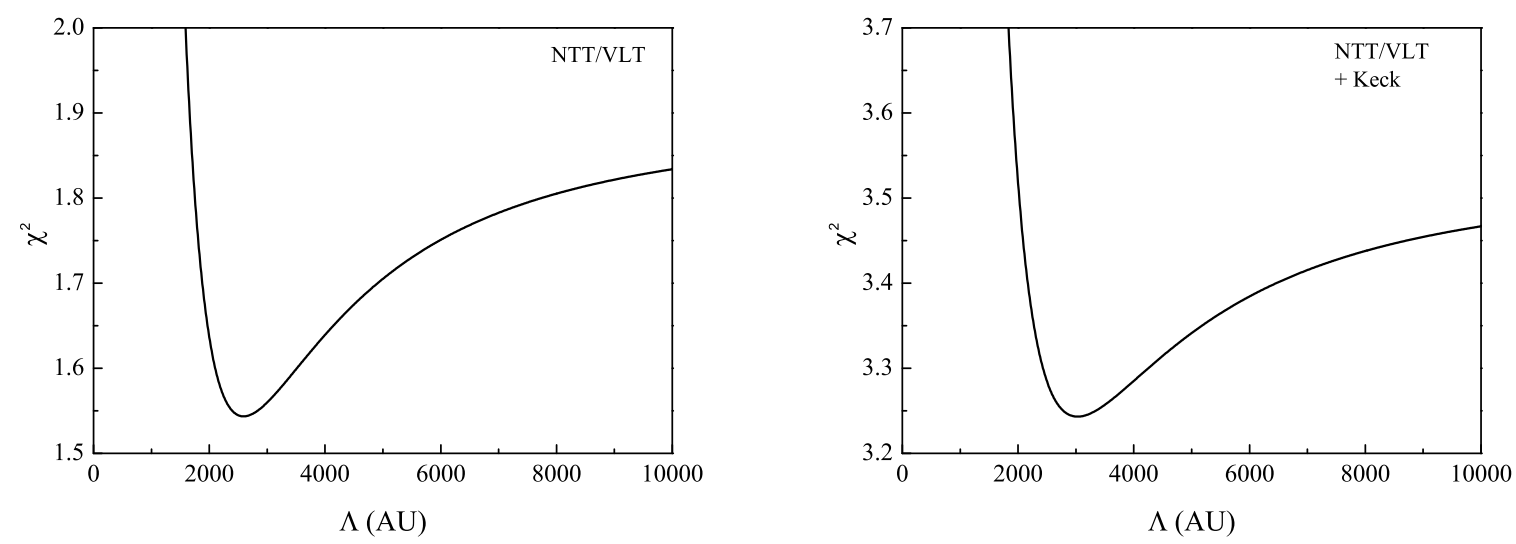

Figure 6. The reduced $\chi^{2}$ for $\delta=1 / 3$ as a function of $\Lambda$ in case of NTT/VLT alone (left) and combined NTT $/$ VLT + Keck (right) observations.
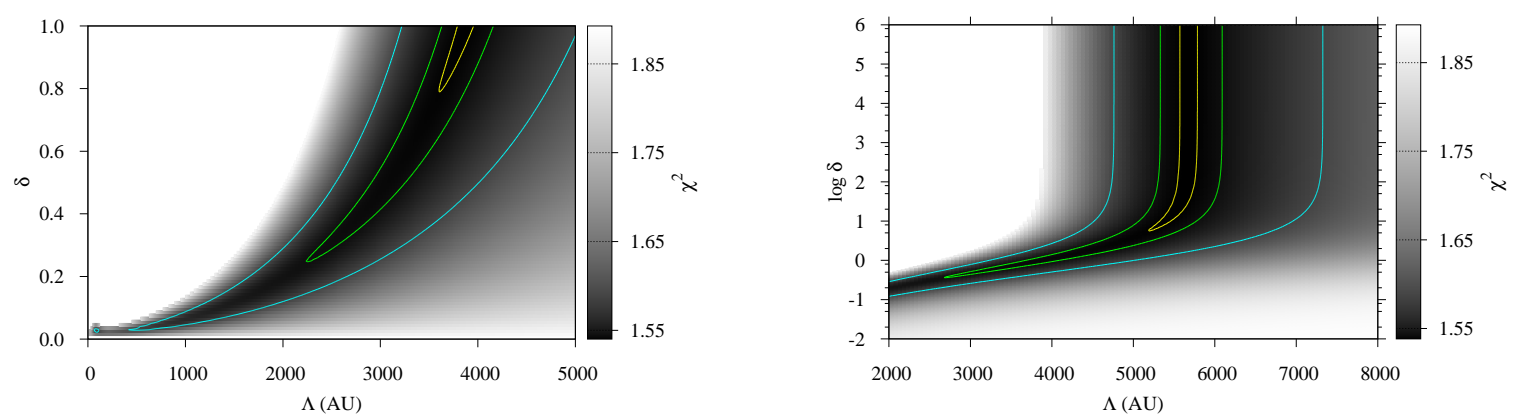

Figure 7. The maps of reduced $\chi^{2}$ over the $\Lambda-\delta$ parameter space in case of NTT/VLT observations. The left panel corresponds to $\delta \in[0,1]$, and the right panel to the extended range of $\delta \in\left[0.01,10^{6}\right]$. The shades of gray color represent the values of the reduced $\chi^{2}$ which are less than the corresponding value in the case of Keplerian orbit, and three contours (from inner to outer) enclose the confidence regions in which the difference between the current and minimum reduced $\chi^{2}$ is less than $0.0005,0.005$ and 0.05 , respectively.
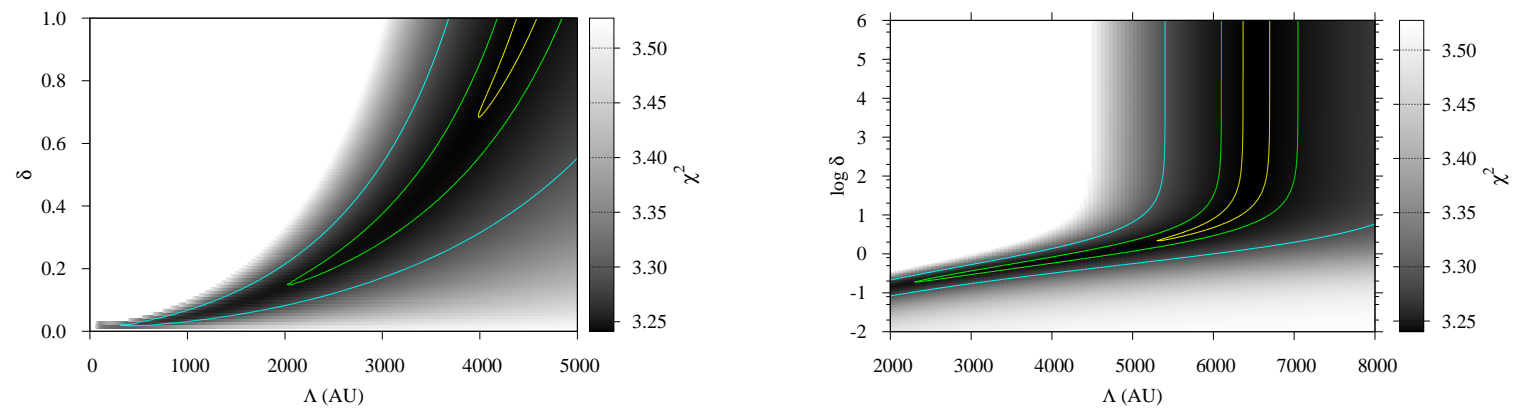

Figure 8. The same as in Fig. 7, but for the combined NTT/VLT + Keck observations.

which even a significant strength of the Yukawa interaction could be expected according to the planetary and Lunar Laser Ranging constraints [44]. Lunar (and artificial satellite) Laser ranging (LLR) is one of the most accurate techniques to test gravitational physics and Einstein's theory of General Relativity [76-78] at the corresponding length scales. In particular, LLR has provided very accurate tests of the strong equivalence principle at the foundations of 
General Relativity and of the weak equivalence principle, at the basis of any metric theory of gravity. Also, it has provided strong limits to the values of the so-called PPN (Parametrized Post-Newtonian) parameters [78]. The Solar System and LLR constraints on the range of Yukawa interaction are shown in Fig. 16 (see also Table 8) in Adelberger et al. [44], according to which $\Lambda \gg 1.5 \times 10^{11} \mathrm{~m}$ and $\Lambda \gg 4 \times 10^{8} \mathrm{~m}$, respectively, and thus is in accordance with our findings.

In Fig. 2 we presented two comparisons between the fitted orbits in Yukawa gravity for $\delta=+1 / 3$ through the astrometric observations of S2 star by NTT/VLT alone (left) and NTT/VLT + Keck combination (right). In order to combine NTT/VLT and Keck data sets, the position of the origin of Keck observations is first shifted by $\Delta x=3.7$ and $\Delta y=4.1$ mas, following the suggestion given in [50]. In the first case the best fit is obtained for $\Lambda=2.59 \times 10^{3}$ $\mathrm{AU}$, resulting with reduced $\chi^{2}=1.54$, and in the second case for $\Lambda=3.03 \times 10^{3}$ AU with reduced $\chi^{2}=3.24$. As one can see from these figures, in both cases there is a good agreement between the theoretical orbits and observations, although the higher value of reduced $\chi^{2}$ in the second case indicates possibly larger positional difference between the two coordinate systems, as also noted in [50]. These figures also show that the simulated orbits of S2 are not closed in vicinity of apocenter, indicating a possible orbital precession.

In Figs. 3 and 4 we presented the comparisons between the observed and fitted coordinates of S2 star and their O-C residuals in the case of NTT/VLT observations, as well as $\mathrm{NTT} / \mathrm{VLT}+$ Keck combined data set, respectively. One can notice that in both cases, O-C residuals are higher in the first part of observing interval (up to the 12 mas) and much less in its second part (less than 2 mas). Due to adopted merit function given by Eq.(3.1), our fitting procedure assigned greater weight to these latter, more precise observations. Also, the O-C residuals are larger in the case of the combined NTT/VLT + Keck observations most likely due to the shift of the origin of the coordinate system, which was necessary in order to get a reasonable fit. That is why we also presented the results for NTT/VLT measurements alone.

We also made the comparisons between the observed and fitted radial velocities of S2 star (see Fig. 5) for NTT/VLT data alone (left) and NTT/VLT+Keck combination (right). In the bottom parts of both panels in Fig. 5 the best fit O-C residuals for radial velocities are also given. As it can be seen from Fig. 5, we also obtained satisfactory agreement between the predicted and observed radial velocities of $\mathrm{S} 2$ star.

Figure 6 presents the reduced $\chi^{2}$ for all fits with fixed value of $\delta=1 / 3$ as a function of the other parameter of Yukawa gravity $\Lambda$ which was varied from 10 to $10000 \mathrm{AU}$. In the case of $\mathrm{NTT} /$ VLT observations the minimum of reduced $\chi^{2}$ is 1.54 and is obtained for $\Lambda=2.59 \times 10^{3}$ $\mathrm{AU}$, while in the case of $\mathrm{NTT} / \mathrm{VLT}+$ Keck combined data set the minimal value of 3.24 is obtained for $\Lambda=3.03 \times 10^{3}$ AU. For both cases the reduced $\chi^{2}$ for Keplerian orbits $(\delta=0)$ are 1.89 and 3.53, respectively, and thus significantly higher than the corresponding minima for $\delta=1 / 3$. This means that Yukawa gravity describes observed data even better than Newtonian gravity and that $\delta=1 / 3$ is valid value at galactic scales.

Figs. 7 and 8 present the maps of the reduced $\chi^{2}$ over the $\Lambda-\delta$ parameter space for all simulated orbits of S2 star which give at least the same or better fits than the Keplerian orbits. These maps are obtained by the same fitting procedure as before. The left panels of both figures correspond to $\delta \in[0,1]$ and $\Lambda[\mathrm{AU}] \in[10,5000]$, and the right panels to the extended range of $\delta \in\left[0.01,10^{6}\right]$ and $\Lambda[\mathrm{AU}] \in[2000,8000]$. Three contours (from inner to outer) enclose the confidence regions in which the difference between the current and minimum reduced $\chi^{2}$ is less than 0.0005, 0.005 and 0.05 , respectively. As it can be seen from Fig. 7, the most probable value for the scale parameter $\Lambda$, in the case of NTT/VLT observations of S2 star, 
is around 5000 - $6000 \mathrm{AU}$, while in the case of NTT/VLT +Keck combined data set (Fig. 8), the most probable value for $\Lambda$ is around 6000 - 7000 AU. In both cases $\chi^{2}$ asymptotically decreases as a function of $\delta$, and hence, it is not possible to obtain reliable constrains on the universal constant $\delta$ of Yukawa gravity. Also, these two parameters $\delta$ and $\Lambda$ are highly correlated in the range $(0<\delta<1)$. For $\delta>2$ (the vertical strips) they are not correlated.

As it could be also seen from left panels of Figs. 7 and 8 , the values $\delta \approx 1 / 3$ result with very good fits for which the reduced $\chi^{2}$ deviate from the minimal value for less than 0.005 (middle contours in both figures). The corresponding values for $\Lambda$ range approximately from 2500 to $3000 \mathrm{AU}$. For $\delta=1 / 3$ we obtained the following values: $\Lambda=2590 \pm 5 \mathrm{AU}$ (NTT/VLT data) and $\Lambda=3030 \pm 5 \mathrm{AU}$ (NTT/VLT + Keck combined data).

Although both observational sets indicate that the orbit of S2 star most likely is not a Keplerian one, the current astrometric limit is not sufficient to unambiguously confirm such a claim. However, the accuracy is constantly improving from around 10 mas during the first part of the observational period, currently reaching around 0.3 mas. We hope that in the future, it will be possible to measure the stellar positions with much better accuracy of $\sim 10 \mu$ as [80].

\section{Conclusions}

In this paper orbit of S2 star has been investigated in the framework of the Yukawa gravity. Using the observed positions of S2 star around the Galactic Centre we constrained the parameters of Yukawa gravity. Our results show that:

1. the most probable value for Yukawa gravity parameter $\Lambda$ in the case of S2 star, is around 5000 - $7000 \mathrm{AU}$ and that the current observations do not enable us to obtain the reliable constraints on the universal constant $\delta$;

2. the same universal constant $\delta$ which was successfully applied to clusters of galaxies $[45,46]$ and rotation curves of spiral galaxies [40] also gives a good agreement in the case of observations of S2 star orbit;

3. the scale parameter of Yukawa gravity in the case of S2 star for $\delta=+1 / 3$ is about: $\Lambda \approx 3000 \pm 1500 \mathrm{AU}$;

4. for vanishing $\delta$, we recover the Keplerian orbit of S2 star;

5. for $\delta=+1 / 3$ there is orbital precession in positive direction like in General Relativity, and for $\delta=-1 / 3$ the precession has negative direction, as in the case of extended mass distribution or in $R^{n}$ gravity [26];

6. the two parameters of Yukawa gravity are highly correlated in the range $(0<\delta<1)$. For $\delta>2$ they are not correlated.

Borka et al. [26] found that $R^{n}$ gravity may not represent a good candidate to solve both the rotation curves problem of spiral galaxies and the orbital precession of S2 star for the same value of the universal constant $\beta$ ( $\beta=0.817$ and $\beta \sim 0.01$, respectively). According to the above results, the opposite conclusion is not eliminated in the case of Yukawa gravity with $\delta=1 / 3$.

The constraints on parameter $\Lambda$ obtained in the present paper are in agreement with the corresponding Solar System and LLR constraints presented by Adelberger et al. [44], 
according to which $\Lambda \gg 1.5 \times 10^{11} \mathrm{~m}$ and $\Lambda \gg 4 \times 10^{8} \mathrm{~m}$, respectively. Also, the constraints on the Yukawa-like modifications of gravitation from Solar System planetary motions showed that one can assume values of the parameter $\Lambda$ on the same order of magnitude, or larger than the typical sizes of the planetary orbits in the Solar System [17]. This is common assumption in many modified theories of gravity $[17,79]$ and is also in accordance with our findings.

However, one should keep in mind that we considered an idealized model ignoring many uncertain factors, such as an extended mass distribution, perturbations from nonsymmetric mass distribution, etc. Therefore, the future observations with advanced facilities, such as GRAVITY which will enable extremely accurate measurements of the positions of stars of $\sim$ $10 \mu$ as [80], or E-ELT with expected accuracy of $\sim 50-100 \mu$ as [81], are needed in order to verify these claims.

\section{Acknowledgments}

This research is part of the project 176003 "Gravitation and the large scale structure of the Universe" supported by the Ministry of Education, Science and Technological Development of the Republic of Serbia. AFZ was supported in part by the NSF (HRD-0833184) and NASA (NNX09AV07A) at NCCU in Durham. The authors thank a referee for a constructive criticism.

\section{References}

[1] E. Fischbach and C. L. Talmadge, The Search for Non-Newtonian Gravity, 305p., Heidelberg-New York, Springer (1999).

[2] T. Clifton, Alternative Theories of Gravity, University of Cambridge (2006).

[3] T. Clifton, P. G. Ferreira, A. Padilla, and C. Skordis, Modified gravity and cosmology, Physics Reports, vol. 513 (2012) 1.

[4] S. Capozziello, and M. de Laurentis, Extended Theories of Gravity, Physics Reports, vol. 509 (2011) 167.

[5] S. Capozziello, V. Faraoni, Beyond Einstein Gravity: A Survey of Gravitational Theories for Cosmology and Astrophysics, Fundamental Theories of Physics vol. 170, Springer (2011).

[6] M. Milgrom, A modification of the Newtonian dynamics as a possible alternative to the hidden mass hypothesis, Astrophys. J. 270 (1983) 365.

[7] M. Milgrom and R. H. Sanders, Modified Newtonian dynamics and the "dearth of dark matter in ordinary elliptical galaxies", Astrophys. J. 599 (2003) L25.

[8] J. D. Bekenstein, Relativistic gravitation theory for the modified Newtonian dynamics paradigm, Phys. Rev. D 70 (2004) 083509; Phys. Rev. D 71 (2005) 069901(E).

[9] J. Bekenstein and J. Magueijo, Modified Newtonian Dynamics habitats within the solar system, Phys. Rev. D 73 (2006) 103513.

[10] C. Brans and H. Dicke, Mach's principle and a relativistic theory of gravitation, Phys. Rev. 124 (1961) 925.

[11] D. Behnke, D. B. Blaschke, V. N. Pervushin, and D. Proskurin, Description of supernova data in conformal cosmology without cosmological constant, Phys. Lett. B 530 (2002) 20.

[12] B. M. Barbashov, V. N. Pervushin, A. F. Zakharov, and V. A. Zinchuk, Hamiltonian cosmological perturbation theory, Phys. Lett. B 633 (2006) 458. 
[13] E. Fischbach, D. Sudarsky, and A. Szafer et al., Reanalysis of the Eoumltvös experiment, Phys. Rev. Lett. 56, (1986) 3; Phys. Rev. Lett. 56 (1986) 1427.

[14] E. Fischbach and C. Talmadge, Six years of the fifth force, Nature 356 (1992) 207.

[15] C. D. Hoyle, U. Schmidt, B. R. Heckel, E. G. Adelberger, J. H. Gundlach, D. J. Kapner, and H. E. Swanson, Submillimeter test of the gravitational inverse-square law: A search for "large" extra dimensions, Phys. Rev. Lett. 86 (2001) 1418.

[16] Z. Berezhiani, F. Nesti, L. Pilo and N. Rossi, Gravity Modification with Yukawa-type Potential: Dark Matter and Mirror Gravity, arXiv:0902.0144v2 (2010).

[17] L. Iorio, Constraints on the range $\Lambda$ of Yukawa-like modifications to the Newtonian inverse-square law of gravitation from Solar System planetary motions, JHEP 10 (2007) 041.

[18] S. Capozziello, Curvature Quintessence, Int. J. Mod. Phys. D 11 (2002) 483.

[19] S. Capozziello, V. F. Cardone, S. Carloni, and A. Troisi, Curvature quintessence matched with observational data, Int. J. Mod. Phys. D 12 (2003) 1969.

[20] S. M. Carroll, V. Duvvuri, M. Trodden, and M. S. Turner, Is cosmic speed-up due to new gravitational physics?, Phys. Rev. D 70 (2004) 043528.

[21] G. Leon and E. N. Saridakis, Dynamics of the anisotropic Kantowsky-Sachs geometries in $R^{n}$ gravity, Class. Quantum Grav. 28 (2011) 065008.

[22] T. P. Sotiriou and V. Faraoni, $f(R)$ theories of gravity, Rev. Mod. Phys. 82 (2010) 451.

[23] S. Capozziello, V. F. Cardone, and A. Troisi, Gravitational lensing in fourth order gravity, Phys. Rev. D 73 (2006) 104019.

[24] S. Capozziello, V. F. Cardone, and A. Troisi, Low surface brightness galaxy rotation curves in the low energy limit of $R^{n}$ gravity: no need for dark matter?, Mon. Not. R. Astron. Soc. 375 (2007) 1423.

[25] S. Kopeikin, I. Vlasov, Parametrized post-Newtonian theory of reference frames, multipolar expansions and equations of motion in the N-body problem, Physics Reports 400 (2004) 209.

[26] D. Borka, P. Jovanović, V. Borka Jovanović, and A. F. Zakharov, Constraints on $R^{n}$ gravity from precession of orbits of S2-like stars, Phys. Rev. D 85 (2012) 124004.

[27] C. Frigerio Martins and P. Salucci, Analysis of rotation curves in the framework of $R^{n}$ gravity, Mon. Not. R. Astron. Soc. 381 (2007) 1103.

[28] A. F. Zakharov, A. A. Nucita, F. De Paolis, and G. Ingrosso, Solar system constraints on $R^{n}$ gravity, Phys. Rev. D 74 (2006) 107101.

[29] A. F. Zakharov, A. A. Nucita, F. De Paolis, and G. Ingrosso, Apoastron shift constraints on dark matter distribution at the Galactic Center, Phys. Rev. D 76 (2007) 062001.

[30] A. A. Nucita, F. De Paolis, G. Ingrosso, A. Qadir, and A. F. Zakharov, Sgr A*: A laboratory to measure the central black hole and stellar cluster parameters, Publ. Astron. Soc. Pac. 119 (2007) 349 .

[31] S. Capozziello, A. Stabile, and A. Troisi, A general solution in the Newtonian limit of $f(R)$ gravity, Mod. Phys. Lett. A 24 (2009) 659.

[32] L. Iorio, Galactic orbital motions in the dark matter, modified Newtonian dynamics and modified gravity scenarios, Mon. Not. R. Astron. Soc. 401 (2010) 2012.

[33] V. A. Rubakov and P. G. Tinyakov, Infrared-modified gravities and massive gravitons, Phys. Usp. 51 (2008) 759.

[34] E. Babichev, C. Deffayet, and R. Ziour, Recovery of general relativity in massive gravity via the Vainshtein mechanism, Phys. Rev. D 82 (2010) 104008. 
[35] J. B. Pitts and W. C. Schieve, Universally coupled massive gravity, Theor. Math. Phys. 151(2) (2007) 700.

[36] E. Babichev, C. Deffayet, and R. Ziour, Recovering General Relativity from Massive Gravity, Phys. Rev. Lett. 103 (2009) 201102.

[37] C. de Rham, G. Gabadadze, and A. J. Tolley, Resummation of massive gravity, Phys. Rev. Lett. 106 (2011) 231101.

[38] Gong Yun-Gui, Cosmology in Massive Gravity, Commun. Theor. Phys. 59 (2013) 319.

[39] E. Babichev and A. Fabbri, Instability of black holes in massive gravity, Class. Quantum Grav. 30 (2013) 152001.

[40] V. F. Cardone and S. Capozziello, Systematic biases on galaxy haloes parameters from Yukawa-like gravitational potentials, Mon. Not. R. Astron. Soc. 414 (2011) 1301.

[41] M. Sereno and Ph. Jetzer, Dark matter versus modifications of the gravitational inverse-square law: results from planetary motion in the Solar system, Mon. Not. R. Astron. Soc. 371 (2006) 626.

[42] C. Talmadge, J.-P. Berthias, R. W. Hellings, and E. M. Standish, Model-independent constraints on possible modifications of Newtonian gravity, Phys. Rev. Lett. 61, No. 10 (1988) 1159.

[43] R. H. Sanders, Anti-gravity and galaxy rotation curves, Astron. Astrophys. 136 (1984) L21.

[44] E. G. Adelberger, J. H. Gundlach, B. R. Heckel, S. Hoedl, and S. Schlamminger, Torsion balance experiments: a low-energy frontier of particle physics, Progress in Particle and Nuclear Physics 62 (2009) 102.

[45] S. Capozziello, A. Stabile, and A. Troisi, Newtonian limit of $f(R)$ gravity, Phys. Rev. D 76 (2007) 104019.

[46] S. Capozziello, E. de Filippis, and V. Salzano, Modelling clusters of galaxies by $f(R)$-gravity, Mon. Not. R. Astron. Soc. 394 (2009) 947.

[47] N. R. Napolitano, S. Capozziello, A. J. Romanowsky, M. Capaccioli, and C. Tortora, Testing Yukawa-like potentials from $f(R)$-gravity in elliptical galaxies, Astrophys. J. 748 (2012) 87.

[48] A. M. Ghez, M. Morris, E. E. Becklin, A. Tanner, and T. Kremenek, The accelerations of stars orbiting the Milky Way's central black hole, Nature 407 (2000) 349.

[49] A. M. Ghez, S. Salim, N. N. Weinberg, J. R. Lu, T. Do, J. K. Dunn, K. Matthews, M. R. Morris, S. Yelda, E. E. Becklin, T. Kremenek, M. Milosavljević, and J. Naiman, Measuring distance and properties of the Milky Way's central supermassive black hole with stellar orbits, Astrophys. J. 689 (2008) 1044.

[50] S. Gillessen, F. Eisenhauer, T. K. Fritz, H. Bartko, K. Dodds-Eden, O. Pfuhl, T. Ott, and R. Genzel, The orbit of the star S2 around SGR A* from very large telescope and Keck data, Astrophys. J. 707 (2009) L114.

[51] S. Gillessen, F. Eisenhauer, S. Trippe, T. Alexander, R. Genzel, F. Martins, and T. Ott, Monitoring stellar orbits around the massive black hole in the Galactic Center, Astrophys. J. 692 (2009) 1075.

[52] R. Schödel, T. Ott, R. Genzel, et al., Closest star seen orbiting the supermassive black hole at the Centre of the Milky Way, Nature 419 (2002) 694.

[53] R. Genzel, F. Eisenhauer, and S. Gillessen, The Galactic Center massive black hole and nuclear star cluster, Rev. Mod. Phys. 82 (2010) 3121.

[54] S. Gillessen, R. Genzel, T. K. Fritz et al., A gas cloud on its way towards the supermassive black hole at the Galactic Centre, Nature 481 (2012) 51. 
[55] L. Meyer, A. M. Ghez, R. Schödel, et al., The Shortest-Known-Period Star Orbiting Our Galaxy's Supermassive Black Hole, Science 338 (2012) 84.

[56] G. F. Rubilar and A. Eckart, Periastron shifts of stellar orbits near the Galactic Center, Astron. Astrophys. 374 (2001) 95.

[57] G. S. Adkins and J. McDonnell, Orbital precession due to central-force perturbations, Phys. Rev. D 75 (2007) 082001.

[58] N. N. Weinberg, M. Milosavljević, and A. M. Ghez, Stellar dynamics at the Galactic Center with an extremely large telescope, Astrophys. J. 622 (2005) 878.

[59] T. Fritz, S. Gillessen, S. Trippe, T. Ott, H. Bartko, O. Pfuhl, K. Dodds-Eden, R. Davies, F. Eisenhauer, R. Genzel, What is limiting near-infrared astrometry in the Galactic Centre?, Mon. Not. R. Astron. Soc. 401 (2010), 1177.

[60] D.E. Krause and E. Fischbach, Searching for extra-dimensions and new string-inspired forces in the Casimir regime, in Gyros, Clocks, Interferometers. . . : Testing Relativistic Gravity in Space, C. Líammerzahl, C.W.F. Everitt and F.W. Hehl eds., Springer-Verlag, Berlin, Germany (2001) [hep-ph/9912276].

[61] O. Bertolami and J. Ptaramos, Astrophysical constraints on scalar field models, Phys. Rev. D 71 (2005) 023521.

[62] O. Bertolami, J. Ptaramos and S. Turyshev, General theory of relativity: will it survive the next decade?, in Lasers, clocks, and drag-free: technologies for future exploration in space and tests of gravity, H. Dittus, C. Líammerzahl and S. Turyshev eds., Springer (2006) [gr-qc/0602016].

[63] J.W. Moffat, Gravitational theory, galaxy rotation curves and cosmology without dark matter, JCAP 05 (2005) 22.

[64] J.W. Moffat, Scalar-tensor-vector gravity theory, JCAP 03 (2006) 004.

[65] M.J. White and C.S. Kochanek, Constraints on the long-range properties of gravity from weak gravitational lensing, Astrophys. J. 560 (2001) 539.

[66] L. Amendola and C. Quercellini, Skewness as a test of the equivalence principle, Phys. Rev. Lett. 92 (2004) 181102.

[67] S. Reynaud and M.-T. Jaekel, Testing the newton law at long distances, Int. J. Mod. Phys. A 20 (2005) 2294.

[68] C. Sealfon, L. Verde and R. Jimenez, Limits on deviations from the inverse-square law on megaparsec scales, Phys. Rev. D 71 (2005) 083004.

[69] A. Shirata, T. Shiromizu, N. Yoshida and Y. Suto, Constraining deviations from Newton $\check{S}$ s law of gravity on cosmological scales: confrontation to power spectrum of SDSS galaxies, Phys. Rev. D 71 (2005) 064030.

[70] R. G. Aitken, "The binary stars" Astronomer in the Lick Observatory, University of California, New York (1918).

[71] W. M. Smart, On the derivation of the elements of a visual binary orbit by Kowalsky's method, Mon. Not. R. Astron. Soc. 90 (1930) 534.

[72] R. H. Sanders, Solar system constraints on multifield theories of modified dynamics, Mon. Not. R. Astron. Soc. 370 (2006) 1519.

[73] L. Iorio, Putting Yukawa-like Modified Gravity (MOG) on the test in the Solar System, Scholarly Research Exchange, Article ID (2008) 238385; arXiv:0809.3563v4 (2008).

[74] M. L. Ruggiero and L. Iorio, Constraining Post-Newtonian $f(R)$ Gravity in the Solar System, arXiv:0811.3860v1 (2008). 
[75] J. J. Moré, B. S. Garbow, and K. E. Hillstrom, User Guide for MINPACK-1, Argonne National Laboratory Report ANL-80-74, Argonne, Ill. (1980).

[76] I. Ciufolini, Frame-dragging, gravitomagnetism and Lunar Laser Ranging, New Astronomy 15 (2010) 332 .

[77] Y. Xie, S. Kopeikin, Post-Newtonian Reference Frames for Advanced Theory of the Lunar Motion and for a New Generation of Lunar Laser Ranging, Acta physica slovaca vol. 60 No. 4 (2010) 393.

[78] I. Ciufolini, A. Paolozzi, E. C. Pavlis, J. Ries, R. Koenig, R. Matzner, G. Sindoni, and H. Neumayer, Testing gravitational physics with satellite laser ranging, Eur. Phys. J. Plus 126 (2011) 72 .

[79] J. W. Moffat, A modified gravity and its consequences for the solar system, astrophysics and cosmology, International Journal of Modern Physics D, 16 (12 A) (2007) 2075.

[80] S. Gillessen, F. Eisenhauer, G. Perrin, et al., GRAVITY spectrometer: metrology laser blocking strategy at $O D=12$, Proc. of SPIE (2010) 77342.

[81] An Expanded View of the Universe: Science with the European Extremely Large Telescope: http://www.eso.org/public/products/brochures/e-eltsciencecase/ 